\title{
Zipper Assembly of Vectorial Rigid-Rod $\pi$-Stack Architectures with Red and Blue Naphthalenediimides: Toward Supramolecular Cascade n/p-Heterojunctions**
}

\author{
Adam L. Sisson, Naomi Sakai, Natalie Banerji, Alexandre Fürstenberg, Eric Vauthey,* and \\ Stefan Matile*
}

In organic optoelectronics and beyond, control of supramolecular organization is essential to create significant functions. ${ }^{[1-6]}$ For this purpose, we have introduced the zipper assembly of rigid-rod $\pi$-stack architectures composed of $p$-oligophenyl (POP) rods and blue naphthalenediimide (NDI) stacks on conducting surfaces. ${ }^{[4]}$ The fact that the observed photocurrent was much higher than that with more conventional layer-by-layer assemblies, and the possibility of terminating the growth of the layers with the component of matching length, demonstrated the existence and the functional significance of the ordered structure in zipper assemblies. These positive results encouraged us to apply this approach and tackle more challenging objectives such as creation of nanoscale $\mathrm{n} / \mathrm{p}$-bulk heterojunctions with wide absorption spectrum. As the first example of multicomponent zipper assembly, we herein report vectorial POP/NDI architectures with $\pi$-stacks composed of red and blue NDIs that promise access to unique, supramolecular ${ }^{[1]} \operatorname{cascade}^{[2]} \mathrm{n} / \mathrm{p}$ heterojunctions, in which redox gradients along coaxial $n-$ and p-semiconductors mediate directional flow of electrons on the molecular level (Figure 1). This approach thus expands the previously reported concept of supramolecular n/p-heterojunctions $^{[1 \mathrm{a}, \mathrm{b}]}$ toward directional cascade architectures and functional nanodevices.

$\mathrm{NDIs}^{[3-7]}$ are perfect modules for zipper assembly because their photo- and electrochemical properties can be easily tuned without global structural changes. ${ }^{[7]}$ Moreover, their

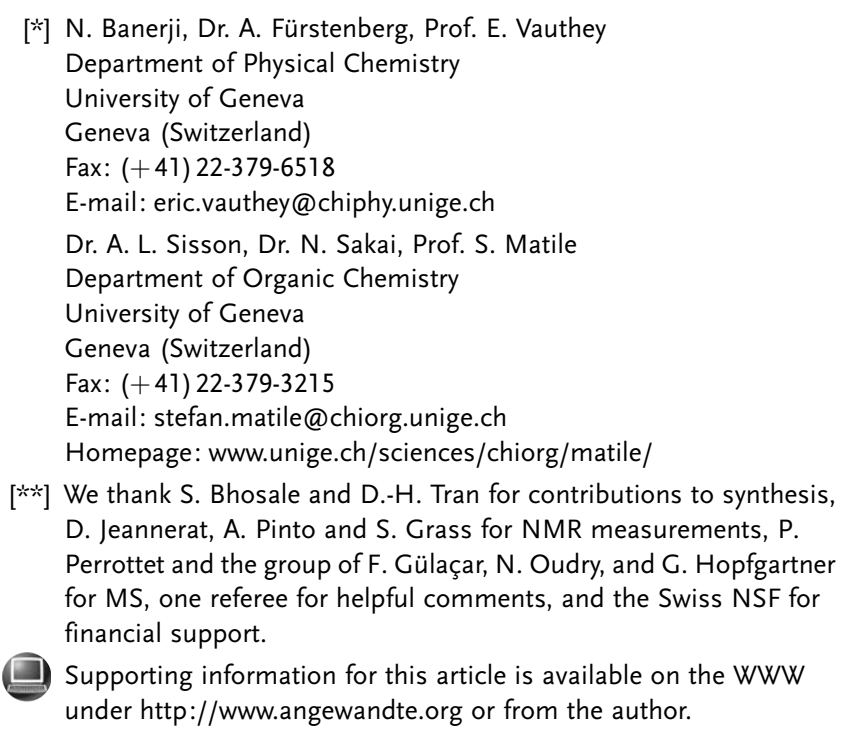

planarity and $\pi$ acidity enables the formation of $n$-semiconducting face-to-face $\pi$ stacks next to strings of p-semiconducting POP rods. ${ }^{[4-6]}$ The expected perpendicular orientation of the resulting stack/rod cascade $\mathrm{n} / \mathrm{p}$-heterojunctions with respect to the surface appeared ideal to secure the elusive high photocurrents in thick chromophore layers needed for efficient absorption ${ }^{[1,2]}$ while maintaining the fill factor (FF) by keeping the resistance low. ${ }^{[8]}$

To assemble multicomponent zippers, such as Au-1-2-3-4, the POP 2 with eight red 2(3)-alkylamino-6(7)-chloro-1,4,5,8NDIs (rNDIs) was synthesized and characterized using procedures developed for blue bNDI POPs 1, 3, and 4 (Figure 1, and Figure S1 and Scheme S1 in the Supporting Information). ${ }^{[4-6]}$ The frontier-orbital energy levels of all zipper components were determined by steady-state fluorescence spectroscopy and cyclic voltammetry (e.g., rNDI monomer: $E_{1 / 2}\left(\mathrm{X} / \mathrm{X}^{+}\right)=+1.21 \mathrm{~V}$ vs $\mathrm{Fc} / \mathrm{Fc}^{+}, E_{1 / 2}\left(\mathrm{X} / \mathrm{X}^{-}\right)=$ $-1.10 \mathrm{~V}$ vs $\mathrm{Fc} / \mathrm{Fc}^{+},{ }^{[\mathrm{bb}]}$ Figure $2 \mathrm{a}$, and Figure S2 and Table S1 in the Supporting Information). To identify the absorption of the $\mathrm{rNDI}^{-}$radical anion, the transient absorption spectra of monomeric Alloc-rNDI 2a (Alloc=allyloxycarbonyl) measured in the absence and the presence of excess $N, N$ dimethylaniline (DMA) were compared (Figure $2 \mathrm{c}, \mathrm{C}$ vs B). Broad bands at $400-510 \mathrm{~nm}$ and $550-700 \mathrm{~nm}$ separated by the ground state bleach were attributed to $\mathrm{rNDI}^{--}$. Although complicated by contributions from the $S_{1}$ state (Figure $2 \mathrm{c}, \mathrm{C}$ ), the presence of these $\mathrm{rNDI}^{-}$bands (Figure $2 \mathrm{c}, \mathrm{B}$ ) in the transient spectra of $\mathbf{2}$ demonstrated the occurrence of photoinduced charge separation (PCS, Figure $2 \mathrm{c}, \mathrm{A}$ ). The decay of this $\mathrm{rNDI}^{--}$revealed that PCS with $\mathbf{2}$ is more than 15 times longer-lived than that with 4 (Figure $2 \mathrm{~d}$ ) ${ }^{[5]}$ Lifetime components reaching beyond $1 \mathrm{~ns}$ were compatible with the stabilization of PCS by intramolecular charge transfer from POPs to rNDIs in $\mathbf{2}$ (Figure $2 \mathrm{a}$, red arrow), although contributions from other origins such as location in the Marcus inverted region remain possible. The observed fluorescence quenching of rNDIs but not bNDIs with $p$ quateranisole or hexamethylbenzene confirmed that photoinduced electron transfer from POP donors is possible to the rNDIs in 2 but not to the bNDIs in 4 (Figure $2 \mathrm{a}$ and $\mathrm{b}$ ). Fluorescence decay kinetics indicated that PCS is complex, ultrafast, and almost quantitative. These findings demonstrated that stack/rod PCS, i.e., the formation of n/p-heterojunctions in zipper assembly, is possible with rNDI 2 but not with bNDI 4. ${ }^{[9]}$

Zipper assembly of Au-1-2-3-4 was initiated by deposition of $p$-quaterphenyl initiators $\mathbf{1}$ on gold electrodes (Figure 1). ${ }^{[4]}$ 


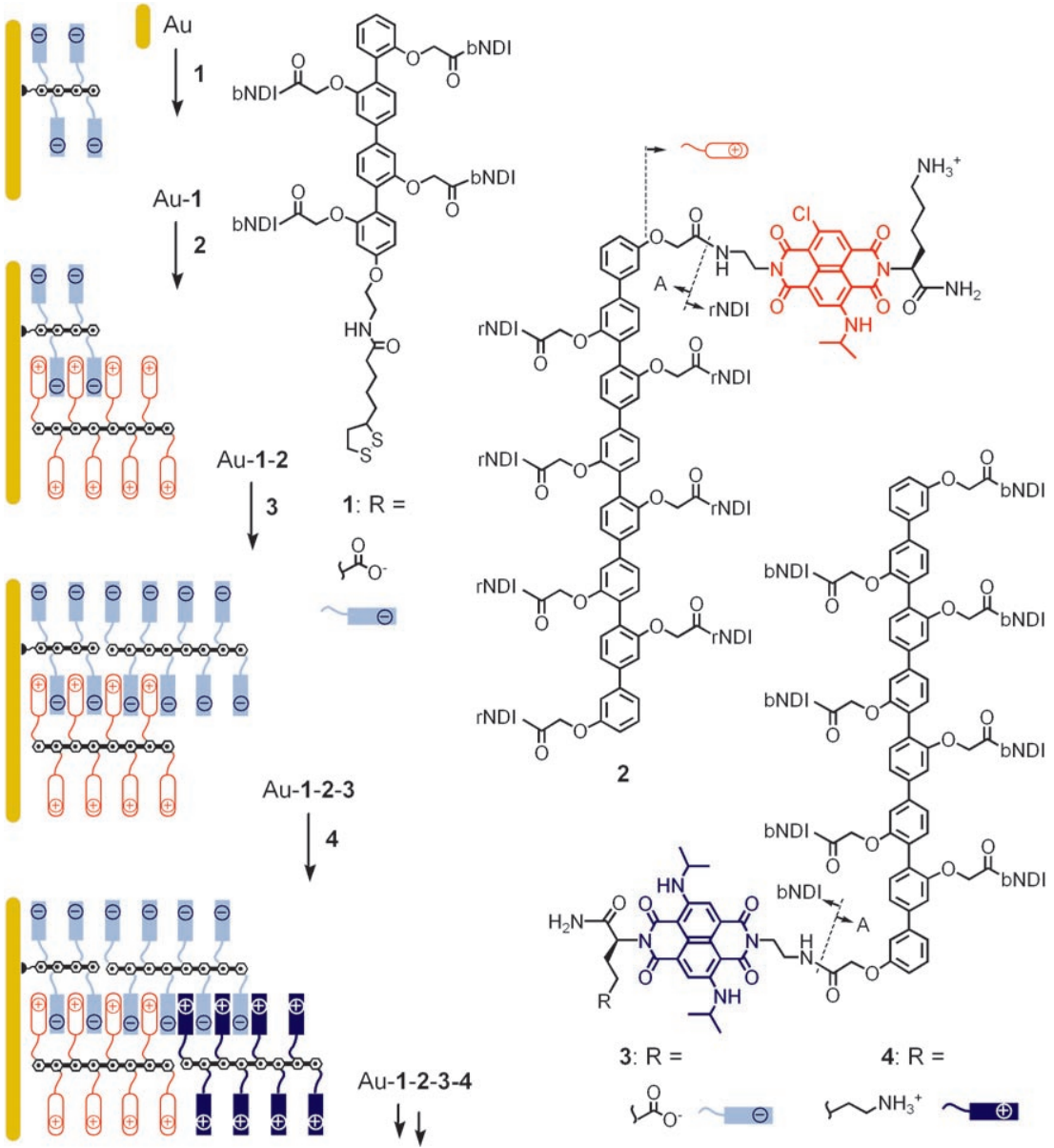

Figure 1. The concept of zipper assembly on gold. All rNDIs are mixtures of 2,6- and 3,7regioisomers. All suprastructures are speculative representations consistent with experimental results ${ }^{[4-6]}$ and molecular models, ${ }^{[5,6]}$ and are shown with the only intention to illustrate the concept. See text for details. 2a, $4 \mathrm{a}: \mathrm{A}=$ Alloc (allyloxycarbonyl). a) $E_{1}$

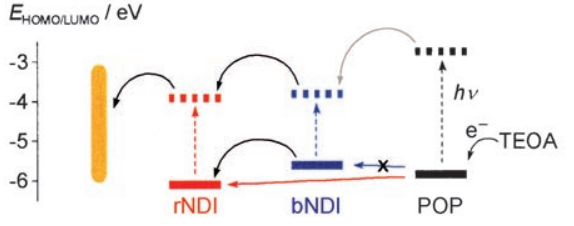

b)

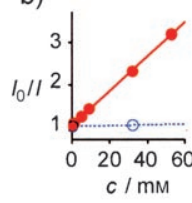

c)

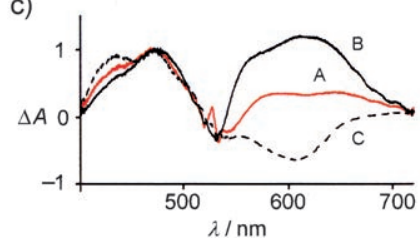

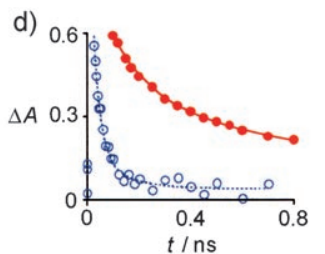

$t / \mathrm{ns}$
Figure 2. a) Zipper energetics with b) evidence for photoinduced stack/rod electron transfer, and c, d) long-lived photoinduced charge separation with 2. a) Frontier orbital energy levels (HOMO, solid; LUMO, dashed) of bNDIs, rNDIs, and POPs. Solid arrows = allowed electron transfer (gray: less likely because of energy transfer), dashed arrows $=$ allowed absorption of light $(h v)$. b) Stern-Volmer plot for quenching of $\mathbf{2 a}(\bullet)$ but not $4 \mathrm{a}(\bigcirc)$ with hexamethylbenzene in acetonitrile. c) Transient absorption spectra of 2 (A) and 2 a (B, 3 ps; $C, 50 \mathrm{ps}$ after a $50 \mathrm{fs}$ laser pulse at $530 \mathrm{~nm}$ ) in $\mathrm{MeOH}$ with (B) or without DMA $(A, C)$. d) Intensity-normalized time profiles of the transient absorption of $4(\bigcirc)$ at $511 \mathrm{~nm}^{[5]}$ and 2 at $470 \mathrm{~nm}(\bullet)$.
The Au-1 obtained was dipped into an aqueous solution of $p$-octiphenyl propagator 2 . Driven by $\pi-\pi$ stacking, intrastack hydrogen bonding, ${ }^{[6]}$ and interstack electrostatic attraction, ${ }^{[4]}$ the lower half of the rNDIs of 2 was expected to $\pi$ stack with the bNDIs of $\mathbf{1}$, whereas the upper half remained available to zip up with anionic $\mathbf{3}$, which in turn can zip up with cationic $\mathbf{4}$. The all-blue $\mathrm{Au}-\mathbf{1 - 4 - 3 - 4}$, structural isomer $\mathrm{Au-1-4-3-2}$, and the doubly red $\mathrm{Au-1-2-3-2}$ were prepared analogously (Figure 3).

Photocurrents were generated by irradiation with light in the presence of triethanolamine (TEOA) as sacrificial electron donor, a gold electrode as electron acceptor, and a platinum electrode as cathode. Current-voltage $(I-V)$ relationships revealed short circuit current $I_{\mathrm{SC}}$, open circuit potential $V_{\text {OC }}$, and the fill factor FF (Figure $3 \mathrm{a}$ ). The $I-V$ profile of the all-blue Au-1-4-3-4 revealed an already good FF of 0.43 (Figure $3 \mathrm{a}, \mathrm{D}$ ). Incorporation of $\mathbf{2}$ strongly increased the $I_{\mathrm{SC}}$. This increase was independent of the directionality of the zipper cascade architecture (Au-1-2-3-4 (B) versus isomer Au1-4-3-2 (C)). However, positioning of rNDI acceptor $\mathbf{2}$ against the flow of electrons in Au-1-4-3-2 did reduce the FF to 0.31 (Figure $3 \mathrm{C}$ ). In clear contrast, the constructive directionality in Au-1-2-3-4 with the rNDI acceptor $\mathbf{2}$ in perfect position to accept electrons for transfer to the gold electrode increased the FF up to 0.55 (Figure $3 \mathrm{a}, \mathrm{B}$ ). Taking $\mathrm{FF}=0.25$ of linear $I-V$ correlations and $\mathrm{FF}=0.61$ of optimized organic solar cells ${ }^{[1 \mathrm{c}]}$ as standard values $(0$ and $100 \%$, respectively), the significant difference in FFs between Au-1-4-3-2 and Au-1-2-3-4 is clearly demonstrated, with $17 \%$ for the former $(\mathrm{FF}=0.31)$ and $90 \%$ for the latter $(\mathrm{FF}=0.55)$. Incorporation of another rNDI acceptor 2 in Au-1-2-3-2 further increased the $I_{\mathrm{SC}}$ because of the increased number of rNDI sensitizers (Figure 3a, A). The FF in Au-1-2-3-2 however decreased to 0.48 because of the weaker redox gradient compared to Au-1-2-3-4. This result confirmed that the adaptable directionality of multicomponent cascade zipper architectures provides access to the rational, molecular-level control of the FF, one of the key factors to generate power with light (Figure $3 \mathrm{a}$ ). The action spectra of these model zippers readily revealed the higher photoactivity of red compared to blue NDIs (Figure $3 \mathrm{~b}$ ). This finding was in agreement with the optoelectronic properties of rNDI POP 2 as described above and supported the formation of supramolecular cascade stack/rod n/p-heterojunctions.

In conclusion, focusing on functional significance, two central, interconnected, but otherwise often elusive key challenges can be addressed with zipper assembly, i.e., supramolecular $\mathrm{n} / \mathrm{p}$-heterojunctions and multicomponent 
a) $\quad \mathrm{V} / \mathrm{V}$ vs $\mathrm{Ag} / \mathrm{AgCl}$
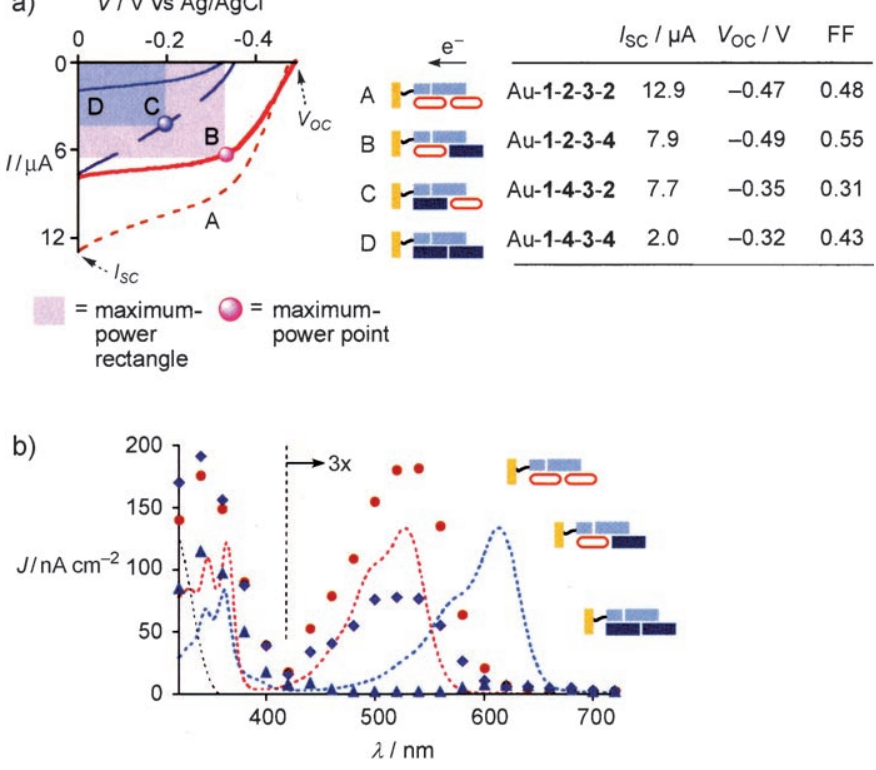

Figure 3. a) Current-voltage profiles with indication of short circuit current $\left(I_{S C}\right)$ and open circuit voltage ( $V_{O C}$, both for Au-1-2-3-2), and maximumpower rectangle and maximum-power point (Au-1-2-3-4 vs Au-1-4-3-2). The fill factor (FF) is defined as maximum power/ $\left(V_{\circ c} I_{s c}\right)$. b) Action

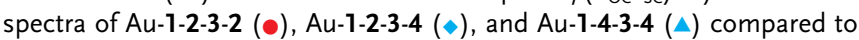
absorption spectra of monomeric bNDIs, rNDIs, and $p$-octiphenyls in $\mathrm{MeOH}$ (dotted blue, pink, and gray lines, respectively).

cascade architecture. Compatibility with supramolecular n/pheterojunctions is demonstrated with photoinduced stack/rod electron transfer from POP donors to the new rNDI acceptors, and reflected in long-lived PCS and high relative photocurrents in current-voltage relationships and action spectra. Functional relevance of the directionality of multicomponent cascade architecture is demonstrated with rational control of the fill factors in current-voltage relationships, that is, the generation of power with light. The perspective to zip in bromo-, alkyloxy-, alkylsulfido-, hydrogen-, or cyano-substituted NDIs ${ }^{[3,7]}$ as variable electron/energy donors/acceptors of variable color in variable patterns promises access to the complexity associated with interesting function, not to speak of the enormous structural and functional scope conceivable with other rods, stacks, and surfaces. With the evidence for significant function in hand, structural and mechanistic studies became more attractive. Collaborative efforts on structural characterization of zipper architectures are ongoing; preliminary results from quartz crystal microbalance and atomic force microscopy are compatible with design and function. However, the complexity of the system calls for caution and suggests that alternative explanations including the existence of different suprastructural packing motifs cannot be excluded despite the availability of a consistent set of experimental data. As far as applications are concerned, other conductive substrates will be needed. Because of its well-characterized surface chemistry, gold is an ideal substrate to determine the importance of multicomponent cascade architectures for function (expressed, for example, in fill factors or relative photocurrents). But it is not an ideal substrate to measure meaningful efficiencies, as evidenced by the increase of photocurrents by a factor of ca. 280 when moving from gold to indium tin oxide electrodes. ${ }^{[10]}$ Efforts toward building zipper assemblies on the other conducting materials are also ongoing.

Received: January 15, 2008

Revised: February 11, 2008

Published online: March 25, 2008

Keywords: artificial photosynthesis - multicomponent reactions . photochemistry · solar energy · supramolecular chemistry

[1] a) F. Würthner, Z. Chen, F. J. M. Hoeben, P. Osswald, C.-C. You, P. Jonkheijm, J. Herrikhuyzen, A. P. H. J. Schenning, P. P. A. M. van der Schoot, E. W. Meijer, E. H. A. Beckers, S. C. J. Meskers, R. A. J. Janssen, J. Am. Chem. Soc. 2004, 126, 10611-10618; b) Y. Yamamoto, T. Fukushima, Y. Suna, N. Ishii, A. Saeki, S. Seki, S. Tagawa, M. Taniguchi, T. Kawai, T. Aida, Science 2006, 314, 1761-1764; c) B. C. Thompson, J. M. J. Fréchet, Angew. Chem. 2008, 120, 62-82, Angew. Chem. Int. Ed. 2008, 47, 58-77.

[2] a) F. B. Abdelrazzaq, R. C. Kwong, M. E. Thompson, J. Am. Chem. Soc. 2002, 124, 4796-4803; b) M. Morisue, S. Yamatsu, N. Haruta, Y. Kobuke, Chem. Eur. J. 2005, 11, 5563-5574; c) D. M. Guldi, J. Phys. Chem. B 2005, 109, 11432-11441.

[3] B. A. Jones, A. Facchetti, M. R. Wasielewski, T. J. Marks, J. Am. Chem. Soc. 2007, 129, 15259-15278.

[4] N. Sakai, A. L. Sisson, T. Bürgi, S. Matile, J. Am. Chem. Soc. 2007, 129, 15758-15759.

[5] S. Bhosale, A. L. Sisson, P. Talukdar, A. Fürstenberg, N. Banerji, E. Vauthey, G. Bollot, J. Mareda, C. Röger, F. Würthner, N. Sakai, S. Matile, Science 2006, 313, 84-86.

[6] P. Talukdar, G. Bollot, J. Mareda, N. Sakai, S. Matile, J. Am. Chem. Soc. 2005, 127, 6528-6529.

[7] a) F. Würthner, S. Ahmed, C. Thalacker, T. Debaerdemaeker, Chem. Eur. J. 2002, 8, 4742-4750; b) C. Thalacker, C. Röger, F. Würthner, J. Org. Chem. 2006, 71, 8098-8105; c) A. Blaszczyk, M. Fischer, C. von Hänisch, M. Mayor, Helv. Chim. Acta 2006, 89, 1986-2005.

[8] D. W. Sievers, V. Shrotriya, Y. Yang, J. Appl. Phys. 2006, 100, $114509-114517$.

[9] The photophysics of these systems is complex, and a comprehensive analysis will be reported in a specialized journal.

[10] H. Yamada, H. Imahori, Y. Nishimura, I. Yamazaki, T. K. Ahn, S. K. Kim, D. Kim, S. Fukuzumi, J. Am. Chem. Soc. 2003, 125, 9129-9139. 